\title{
The Fatty liver Index (FLI) 15 years later: a reappraisal
}

\author{
Amedeo Lonardo ${ }^{1}$, Stefano Ballestri ${ }^{2}$, Giorgio Bedogni ${ }^{3}$, Stefano Bellentani ${ }^{3}$, Claudio Tiribelli ${ }^{3}$ \\ 'Ospedale Civile di Baggiovara, Azienda Ospedaliero-Universitaria, Modena 41126, Italy. \\ ${ }^{2}$ Ospedale di Pavullo, Azienda Ospedaliera, Modena 22037, Italy. \\ ${ }^{3}$ Fondazione Italiana Fegato, Basovizza, Trieste 34149, Italy.
}

Correspondence to: Amedeo Lonardo, MD, Ospedale Civile di Baggiovara, Azienda Ospedaliero-Universitaria, Via Giardini 1135, Modena 41126, Italy. E-mail: a.Ionardo@libero.it; lonardo.amedeo@aou.mo.it

How to cite this article: Lonardo A, Ballestri S, Bedogni G, Bellentani S, Tiribelli C. The Fatty liver Index (FLI) 15 years later: a reappraisal. Metab Target Organ Damage 2021;1:10. https://dx.doi.org/10.20517/mtod.2021.08

Received: 9 Aug 2021 First Decision: 25 Oct 2021 Revised: 1 Nov 2021 Accepted: 15 Nov 2021 Published: 19 Nov 2021

Academic Editor: Águeda González-Rodríguez Copy Editor: Xi-Jun Chen Production Editor: Xi-Jun Chen

\begin{abstract}
The Fatty Liver Index (FLI) is a non-invasive biomarker proposed, in 2006, by Bedogni's group, to aid in identifying patients with suspected nonalcoholic fatty liver disease (NAFLD) to be submitted to liver ultrasonography to confirm steatosis. Criteria of Assessment of Narrative Review Articles, a scale for the assessment of quality of narrative review articles, inspired our review article, which aims at evaluating the scope of published articles on FLI issued over the last 15-year period. The analysis of retrieved data identified the following conclusions. First, given that FLI and NAFLD share the same risk factors, FLI can be used to identify NAFLD among populations at risk to be submitted to screening. Second, FLI is able to identify the hazard of atherosclerosis, both at a subclinical stage and as an overt disease. Third, FLI detects incident diabetes and chronic kidney disease. However, evidence supporting the notion that FLI also predicts the metabolic syndrome, some endocrine disorders, certain tumor types, and overall and cause-specific mortality appears to be more limited. In conclusion, 15 years after its first publication, FLI has been validated as a robust biomarker of both steatosis and NAFLD. Moreover, the scope of FLI has been expanded to previously unexpected areas. Finally, we discuss FLI limitations and a research agenda aimed at further improving the accuracy of FLI scores in predicting liver-related outcomes, endocrine-metabolic disorders, cancer risk, and survival.
\end{abstract}


Keywords: Atherosclerosis, chronic kidney disease, endocrine disorders, metabolic syndrome, mortality, nonalcoholic fatty liver disease, tumors, type 2 diabetes mellitus

\section{BACKGROUND AND AIMS}

\section{Nonalcoholic fatty liver disease: definition and natural course of hepatic and extra-hepatic involvement}

Nonalcoholic fatty liver disease (NAFLD) describes hepatic fatty changes which are bi-directionally associated with the metabolic syndrome (MetS) and its individual components ${ }^{[1]}$. By definition, NAFLD requires the exclusion of competing causes of liver disease.

As a systemic disorder, NAFLD has a "hepatic" as well as an "extra-hepatic" natural history. The former includes manifestations such as simple steatosis, nonalcoholic steatohepatitis (NASH), fibrosis, cirrhosis, and hepatocellular carcinoma ${ }^{[2,3]}$. The latter comprises involvement of the cardiovascular and endocrine systems, chronic respiratory disorders, the musculoskeletal system, the skin, and extra-hepatic tumors ${ }^{[4]}$. Recently, NAFLD has also been clearly identified as a strong risk factor for incident chronic kidney disease $(\mathrm{CKD})^{[5]}$.

According to the European Association for the Study of the Liver, further to ultrasonography, assessment of steatosis can also be accomplished with biomarkers such as the Fatty Liver Index (FLI), SteatoTest, and NAFLD Fat score ${ }^{[6]}$.

\section{A brief history of FLI development and original aims}

In 2006, Bedogni et al. ${ }^{[7]}$ published an article entitled: "The Fatty Liver Index: a simple and accurate predictor of hepatic steatosis in the general population". This study validated an algorithm, based on body mass index (BMI), waist circumference, triglycerides, and gamma-glutamyltransferase (GGT), originally developed based on the analysis of 216 individuals with suspected liver disease compared to 280 controls without liver disease belonging to the general population of the Dionysos Nutrition \& Liver Study (DNLS) ${ }^{[8]}$.

FLI, with a range between 0 and 100, can be calculated using the following two steps.

Step 1. Calculate the linear predictor (LP):

$\mathrm{LP}=0.953 \cdot \ln [$ triglycerides $(\mathrm{mg} / \mathrm{dL})]+0.139 \pm \mathrm{BMI}\left(\mathrm{kg} / \mathrm{m}^{2}\right)+0.718 \cdot \ln [\mathrm{GGT}(\mathrm{IU} / \mathrm{L})]+0.053 \cdot \mathrm{waist}$ circumference $(\mathrm{cm})-15.745$

Step 2. Calculate the probability of fatty liver and multiply it by 100 :

$\mathrm{FLI}=\left[\mathrm{e}^{\mathrm{LP}} /\left(1+\mathrm{e}^{\mathrm{LP}}\right)\right] \times 100$

In is the natural logarithm and e is the base of natural numbers. FLI can be calculated using many freely available online calculators, for example the MdCalculator (https://www.mdcalc.com/fatty-liver-index).

In the DNLS, values of FLI < 30 ruled out (negative likelihood ratio $=0.2$ ) and values $\geq 60$ ruled in (positive likelihood ratio $=4.3)$ fatty liver with a discrimination of $0.84(95 \% \mathrm{CI}$ : $0.81-0.87)$ as detected by the area under the receiver operating characteristic curve ${ }^{[7]}$. It is important to cite the precise words used by Bedogni et al. ${ }^{[7]}$ to explain what role they attributed to FLI: "FLI may help physicians to select subjects for liver ultrasonography and intensified lifestyle counseling, and researchers to select patients for epidemiologic studies". As reported in this article, however, this prediction has largely been overcome by an extraordinary 
number of publications using FLI for purposes quite different from those originally suggested by the authors.

\section{How the components of the FLI algorithm fit in the natural course of hepatic and extra-hepatic NAFLD}

Bedogni et al. ${ }^{[8]}$ used data from the DNLS to develop the FLI algorithm for the prediction of fatty liver. Age, sex, and alcohol were not associated with steatosis in any of the multivariable models leading to the development of FLI. The fact that BMI, waist circumference, GGT, and triglycerides were the independent predictors chosen for the final prediction model is in full agreement with our current understanding of the role of overall and regional adiposity ${ }^{[9]}$, lipid phenotype ${ }^{[10]}$, and GGT as an accurate surrogate index of insulin resistance in NAFLD ${ }^{[1]}$. Unfortunately, the role of GGT as a predictor of fatty liver seems to have generally been neglected by American and British physicians, possibly because GGT is not considered a primary liver test in these countries.

\section{Scale for the quality Assessment of Narrative Review Articles}

The present review article adheres to the Scale for the quality Assessment of Narrative Review Articles (SANRA) ${ }^{[12]}$. Without covering originality, topicality, conflicts of interest, or plausibility, and although not designed to provide a precise estimate of the quality of all theoretically possible manuscripts, this scale is based on formal criteria intended to help editors, reviewers, and readers in assessing the quality of a given narrative review article ${ }^{[12]}$. To this end, SANRA utilizes a simple sum scoring system based on quite limited scoring options $(0,1 \text {, and } 2)^{[12]}$. In short, SANRA supports six qualifying points including: (1) justification of the article's importance for each journal's readership; (2) statement of concrete (single or multiple) aims or formulation of questions; (3) transparency about the sources of information on which the text is based and accurate description of search history; (4) extensive backing key statements with adequate referencing to key statements; (5) introducing appropriate arguments underlying scientific reasoning; and (6) appropriate presentation of data ${ }^{[12]}$.

\section{Bibliographic research strategy and aims}

The PubMed database was searched using those articles, without any language restrictions, exhibiting "Fatty Liver Index" in their titles. The search was completed on 1 June 2021. Overall, 93 articles were retrieved, 43 of which were retained based on the agreement of the authors and 50 were deemed as out of the aims of the present study. The reasons for exclusion were studies either based on limited case series or not relevant to illustrate the main topic of the present study, which aims to report on the scope of FLI use in contemporary medical literature.

\section{RISK FACTORS FOR FLI}

Five studies published thus far, as summarized in Table 1, have addressed the risk factors predicting FLI, considered to be a surrogate marker of NAFLD ${ }^{[13-17]}$.

Collectively, the data suggest that, in European and Japanese populations, age, sex, and lifestyle habits, including dietary and sedentary behavior, modulate total and visceral obesity and affect insulin resistance thereby contributing to determining the risk of NAFLD as assessed by FLI [Table 1]. Integrating the conclusions of the original FLI paper and in agreement with general and recent notions pertaining to the epidemiology of NAFLD ${ }^{[18,19]}$, age and sex were identified as important modifiers of FLI variability in these populations ${ }^{[14]}$. The finding that consumption of sweetened beverages predisposes to while eating fruit protects from $\mathrm{FLI}^{[13]}$ is also consistent with studies conducted on NAFLD ${ }^{[20]}$. Finally, the study by Klisic et al. ${ }^{[15]}$ also confirmed that the range of "normal" transaminases must be updated, as originally suggested by Prati et al. ${ }^{[21]}$, and that risk factors for the development of NAFLD vary in adult men and 
assessment of alcohol use, smoking, adiposity,

and physical activity such that higher scores

indicated a more unhealthy lifestyle Tien et al. ${ }^{[17]} 2021 \quad \begin{aligned} & \text { In total, } 1588 \text { Japanese adults (789 men and } 799 \\ & \text { women) were enrolled participating in the Japan nutrient patterns were extracted: Factor } 1 \text { (vitamins, dietary fiber, iron, and potassium }\end{aligned}$ Multi-Institutional Collaborative Cohort Study, B2, and low carbohydrate pattern); and Factor 4 (sodium, protein, and vitamin D pattern) Tokushima Prefecture

Participants were receiving health checkups, employees of local companies, or volunteers

After adjustment for sex, age, and other potential confounding factors, higher Factor 1 scores were significantly associated with lower ORs of NAFLD $(P$ for trend $<0.05)$

Mediated by reduced overall and abdominal adiposity, a diet rich in vitamins, fiber, iron, and potassium was

Factor analysis was applied to energy-adjusted There were significant inverse associations between Factor 1 scores and high BMI and large WC intake of 21 nutrients, and nutrient patterns were extracted

Multiple LRA was used to analyze the

relationships between nutrient patterns and $\mathrm{FLI} \geq$

${ }^{*}$ FLI was calculated in all studies summarized as described in A brief history of FLI development and original aims. ALT: Alanine-aminotransferase; AUC: area under the curve; BMI: body mass index; GDS: German diabetes study; IR: insulin resistance; LRA: logistic regression analysis; ORs: odds ratios; ROC: receiver operating characteristic; SSB: sugar sweetened beverages; SAT: subcutaneous adipose tissue; SUA: serum uric acid; T2D: type 2 diabetes; VAT: visceral adipose tissue; WC: waist circumference.

women and in boys and girls ${ }^{[19]}$.

\section{IDENTIFYING NAFLD AND METABOLIC SYNDROME WITH FLI}

\section{FLI and NAFLD}

The methodological criteria useful to evaluate the accuracy of FLI at identifying fatty liver and more specifically NAFLD have been discussed elsewhere ${ }^{[22]}$. Seven published studies thus far have evaluated the capacity of FLI to detect NAFLD in various epidemiological scenarios [Table 2] ${ }^{[23-29]}$.

Collectively, the studies summarized in Table 2 indicate that, while being a useful tool for evaluating NAFLD in high-risk populations [e.g., type 2 diabetes $(\mathrm{T} 2 \mathrm{D})$ and obstructive sleep apnea], in the individual patient, FLI has a limited liability of ruling in or out NAFLD ${ }^{[2]}$. This conclusion is in agreement with the original report by Bedogni et al..$^{[7]}$ and with the known limitations of prediction algorithms employed at the individual level ${ }^{[30]}$. Moreover, it suggests that FLI should be used for epidemiological rather than clinical purposes. In this connection, Fedchuk et al. ${ }^{[3]}$ compared the performance and limitations of various biomarkers, FLI included, as related to the accepted NAFLD diagnostic standard, i.e., liver histology. It was found that, albeit being able to identify steatosis and insulin resistance, all non-invasive biomarkers had a limited clinical utility given that they are confounded by fibrosis and inflammation and do not accurately quantify fatty changes. It should be noted, however, that FLI was developed in the general population, and this should be taken into account when it is used for prediction purposes ${ }^{[22]}$. 
Table 2. FLI as a detector of NAFLD across various epidemiological scenarios

\begin{tabular}{|c|c|c|}
\hline $\begin{array}{l}\text { Author, year } \\
\text { [Ref.] }\end{array}$ & Method & Findings \\
\hline Klisic et al. $^{[23]} 2018$ & $\begin{array}{l}139 \text { T2D patients ( } 50.1 \% \text { men) were cross-sectionally evaluated } \\
\text { Anthropometric, blood pressure, and biochemical parameters were } \\
\text { recorded }\end{array}$ & 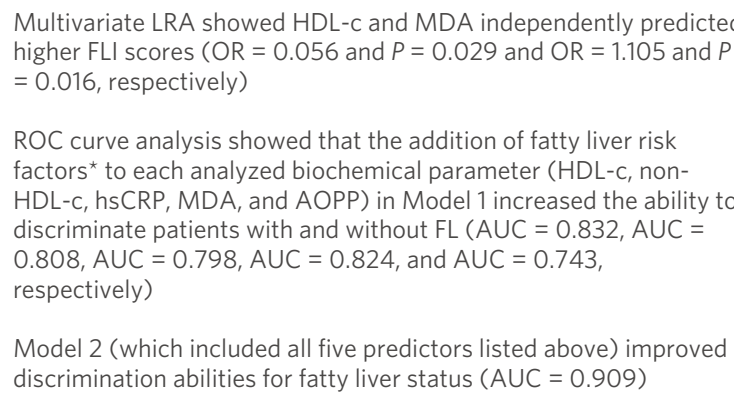 \\
\hline
\end{tabular}

Chen et al. ${ }^{[24]} 2019326$ consecutive adults with and 105 without NAFLD were recruited All were newly diagnosed with OSAHS

Steatosis was diagnosed with US

Accuracy and cutoffs of the FLI and HSI in detecting NAFLD were assessed with AUROC curve and the maximum Youden index analysis, respectively

Hsu et al. ${ }^{[25]} 2019$ From 9293 examinees who underwent routine health checkups, 4000 were enrolled, aged $\geq 20$ years, with a BMI $<24 \mathrm{~kg} / \mathrm{m}^{2}$ in our lean-NAFLD study population. NAFLD diagnoses were made according to the patients histories, laboratory values, and US criteria. Clinical variables, FPG, lipid, and liver profiles were evaluated using multiple LRA. The predictive ability and optimal cutoff values for NAFLD were determined according to the area under the ROC curve

Rabbitt et al. ${ }^{[26]} \quad$ Patients attending the AMU over a 3-month period were invited to

2020 participate. Those with excess alcohol consumption or pre-existing liver participate. Those with

Using established FLI cutoffs, 414 participants were grouped into low (FLI 30 ), medium $(30<\mathrm{FLI} \leq 60)$, and high (FLI $>60$ ) risk of NAFLD

High-risk patients were offered review including LSM and CAP score discrimination abilities for fatty liver status $(A \cup C=0.909)$

Additionally, Model 2 had both higher sensitivity and higher specificity ( $89.3 \%$ and $87.5 \%$, respectively) than each individual predictor in Model 1

Both FLI and HSI values were significantly higher in patients with NAFLD than in controls

The AUROC of FLI and HSI for predicting NAFLD was 0.802 (95\%Cl: 0.762-0.839) and 0.753 (95\% Cl: 0.710-0.793), respectively

FLI had a significantly higher AUROC than $\mathrm{HSI}(P=0.0383)$

The optimal cutoff value of FLI and HSI was 60 (sensitivity $66 \%$ and specificity $80 \%$ ) and 35 (sensitivity $81 \%$ and specificity $60 \%$ ) Overall, $18.5 \%(n=740)$ of the lean population had NAFLD. Male sex, BMI, body fat mass, FPG, SUA, ALT, TG, and FLI values were associated with NAFLD. FLI had the best discriminative ability to predict lean-NAFLD compared to the other biochemical markers. Using the Youden index test, an optimum cutoff value for FLI of 15 was found to have the highest discriminant ability

Conclusion

D patients at a high risk of fatty liver disease may be identified through a structured approach, including biomarkers of oxidative stress, dyslipidemia, and inflammation

In total, 134 patients were at low risk, 96 at medium risk, and 184 at high risk of NAFLD. Male sex $(P<0.0001)$ and increasing age $(P<$ 0.0001 ) were associated with higher risk. Of the 120 high-risk patients who attended follow up, 13 participants had LSM $>7 \mathrm{kPa}$. Higher FLI scores were associated with higher CAP scores $(P<$ 0.0001 ) but did not predict higher LSMs. FGP and HbA1c were found to be associated with higher LSM
Both FLI and $\mathrm{HSI}$ can serve as screening tools for NAFLD in adults with OSAHS

FLI performs better than HSI to this end

The prevalence of lean-NAFLD was not low. sex, liver function, and other metabolic factors, in the prediction of lean-NAFLD. FL may be considered an easy to use, noninvasive marker to screen for lean-NAFLD

About $44.4 \%$ of patients presenting to the AMU were at high risk of NAFLD according to the FLI

Only $10.8 \%$ of the high-risk group and $3 \%$ of all those recruited had a $L S M>7 \mathrm{kPa}$ suggesting development of fibrosis FLI was superior to other predictors including 
Chen et al. ${ }^{[27]} 2020$ Community-based study conducted in Taiwan

Participants were subjected to a demographic survey, blood tests and abdominal US

Motamed et al. ${ }^{[28]}$ This population-based study was based on the results of follow-up on $2020 \quad$ individuals who did not have NAFLD during 2009-2010 but acquired the disease by 2016-2017 in northern Iran

In total, 2241 NAFLD-free individuals at the baseline evaluation in 20092010 were evaluated 7 years later by US to identify incident NAFLD cases

FLI was calculated based on data from Phase 1 (performed in 2009-2010) of the cohort study

ROC analyses were performed to estimate the predictive ability of FLI in diagnosing incident NAFLD cases

In LRA, FLI was considered the predictor and incident NAFLD was the outcome

Castellana et al. ${ }^{[29]}$ Four databases (PubMed, CENTRAL, Scopus, and Web of Science) were 2021 searched until January 2021. Original articles reporting the performance FLI and using US, CT, or MR as a reference standard were included. The numbers of subjects with NAFLD in FLI classes $<30,30-60$, and $\geq 60$, an the numbers of subjects classified as true/false positive/negative when adopting 30 and 60 as cutoffs were extracted. A random-effects model was used for pooling dat
746 individuals were classified in NAFLD group (mean age 56.3) years and 625 controls (average age 57.1)

The optimal cutoff points of FLI to discriminate FL by abdominal US were 20 in male and 10 in female (sensitivity $80.3 \%$ and $76.1 \%$ respectively; specificity $66.9 \%$ and $65.5 \%$, respectively)

FU was correlated with the severity of US FL, predicted fat component percent and NAFLD fibrosis score, particularly in women

AUCs for FLI in men and women were 0.712 (95\% Cl: 0.675-0.749) FLI was able to predict incident NAFLD cases and 0.721 (95\% Cl: 0.683-0.759), respectively

FL was significantly associated with incident NAFLD in LRA in both men and women [OR $(95 \% \mathrm{Cl})=1.038(1.029-1.047), P$-value $<$ 0.001 in men and $\mathrm{OR}(95 \% \mathrm{Cl})=1.032(1.023-1.041)$, P-value $<$ 0.001 in women in multiple]
FLI can be used to select individuals to be submitted to abdominal US in population

To increase sensitivity the FLI threshold might be set at 10 in women and 20 for men
Ten studies, globally evaluating 27,221 subjects without secondary causes of $\mathrm{FL}$, were included

The NAFLD prevalence in the three FLI classes was $14 \%, 42 \%$, and $67 \%$. Sensitivity, specificity, PPV, NPV, LR for positive results, LR for negative results, and diagnostic OR were $81 \%, 65 \%, 53 \%, 84 \%$ $2.3,0.3$, and 7.8 for the lower cutoff and $44 \%, 90 \%, 67 \%, 76 \%$, $4.3,0.6$, and 7.3 for the higher cutoff, respectively. A similar performance was generally found in studies adopting US vs, other

imaging modalities
FLI had an adequate performance in stratifying the risk of NAFLD. However, it showed only weak discriminatory disorder

*Age, sex, body weight, smoking, T2D duration and drugs metabolized in liver. ALT: Alanine transaminase; AMU: acute medical unit; AOPP: advanced oxidant protein products; AUC: area under the curve; AUROC: area under the receiver operating characteristic; BMI: body mass index; CAP: controlled attenuation parameter; Cl: confidence interval; CT: computed tomography; FL: fatty liver; FPG: fasting plasma glucose; HDL-c: high density lipoprotein cholesterol; HSI: hepatic steatosis index; hSCRP: high sensitivity C-reactive protein; LR: likelihood ratio; LRA: logistic regression analysis; LSM: liver stiffness measurement; MDA: malondialdehyde; MR: magnetic resonance; NPV: negative predictive value; OR: odds ratio; OSAHS: obstructive sleep apnea hypopnea syndrome; PPV: positive predictive value; ROC: receiver operating characteristic; SUA: serum uric acid; TG: triglycerides; US: ultrasonography

\section{FLI and the metabolic syndrome}

It should be preliminarily noted that measurement of waist circumference and triglyceride serum concentration are included both in FLI and in MetS. Therefore, an agreement between FLI and MetS, in principle, has to be expected. Two publications have evaluated the association of FLI with the MetS. 
In the first study, Khang et al. ${ }^{[32]}$ evaluated the association between FLI and metabolic disorders and determined the cutoff value of FLI to screen for MetS. To this end, 10,107 adults aged $\geq 19$ years from the Korean National Health and Nutrition Examination Surveys were selected. NAFLD, which was identified based on an increased FLI ( $\geq 60$ ), after the exclusion of alcohol or viral liver disease, had an agestandardized prevalence $=10.0 \%$. Individuals with the higher FLI scores had a higher prevalence of arterial hypertension, T2D, and MetS. At multivariate analysis, the group with higher FLI scores had a significantly higher risk for hypertension ( $\mathrm{OR}=2.92,95 \% \mathrm{CI}: 2.18-3.90, P<0.001)$, T2D $(\mathrm{OR}=4.38,95 \% \mathrm{CI}: 2.96-6.49, P<$ $0.001)$, and MetS (OR $=24.85,95 \% \mathrm{CI}: 17.33-35.64, P<0.001)$. The FLI cutoff value estimated to predict the presence of MetS was 20 (area under the curve 0.849 , sensitivity 0.828 , and negative predictive value $91.9 \%$ ), suggesting that FLI might be employed as a screening tool to identify those individuals in need of early management of MetS.

The second study, by Lee et al. ${ }^{[33]}$, enrolled 3936 women under care at Pusan National University Hospital Health Promotion Center from 2008 to 2014. The overall prevalence of the MetS was 11.6\% with a wide variability based on menopausal status (pre-menopausal 7.0\% and post-menopausal $14.6 \%$ ). The area under the curve of the receiver operating characteristic curve of FLI was 0.93 among pre-menopausal and 0.88 among post-menopausal women, suggesting that FLI should be more carefully applied to post-menopausal women.

\section{FLI vs. US-FLI}

In 2012, by combining the ultrasonographic features of steatosis into a simple semi-quantitative index, Ballestri et al. ${ }^{[34]}$ proposed the so-called "Ultrasonographic Fatty Liver Index" (US-FLI), which was shown to be significantly correlated with metabolic derangements and individual criteria for the histological diagnosis of NASH. Visual examples of the elementary components of ultrasonographic semeiotics of US-FLI have been published elsewhere. In short, the US-FLI scoring system ranges 2-8 based on the intensity of liver/kidney contrast, posterior attenuation of ultrasound beam, vessel blurring, difficult visualization of gallbladder wall, difficult visualization of the diaphragm, and areas of focal sparing. NAFLD is diagnosed by the minimum score 2. US-FLI, initially proposed to select which NAFLD patients should be submitted to liver biopsy, has been validated (reviewed $\mathrm{in}^{[35,36]}$ ). Therefore, it is logical to ascertain whether FLI and USFLI provide comparable clinical information. To answer this research question, Xavier et al. ${ }^{[3]}$ enrolled 96 NAFLD patients in whom transient elastography was performed. They demonstrated that US-FLI was significantly superior to the FLI scores in discriminating between different grades of steatosis, but that the two scores should be applied together to obtain a more precise diagnosis of fatty liver and NAFLD.

\section{DOES FLI GAUGE CARDIOVASCULAR RISK?}

Nine studies from Europe, Asia, and the US evaluated the ability of FLI to identify surrogate indexes of subclinical atherosclerosis and clinically relevant cardiovascular events, as summarized in Table $3^{[38-46]}$.

The data reported in Table 3 consistently show that high FLI scores predict both subclinical atherosclerosis (intracranial vertebrobasilar stenosis, arterial stiffness, and left ventricle mass) and overt disease (incident cardiovascular disease, cardiometabolic disease, heart failure, and adverse major cardiovascular events). These studies are in full agreement with common notions on NAFLD being associated with subclinical atherosclerosis and cardiovascular event ${ }^{[1,47-50]}$. Collectively, studies suggest that, at least for epidemiological purposes, FLI is a reliable marker of the full pre-clinical and clinical spectrum of atherosclerosis at various anatomic sites. 
Table 3. FLI and cardiovascular risk

Author, year Method Findings Conclusion

Qiu et al. ${ }^{[38]} 2017$ A cohort of 2281 Chinese adults recruited from the Wuxi center PMMJS, which was established to analyze the epidemiologic

At the baseline, $\mathrm{FLI}$ was positively associated with prevalent IVBS, and,
compared to the participants with $\mathrm{FLI}<30$, the adjusted $\mathrm{ORs}(95 \% \mathrm{Cl})$ of baseline were enrolled in the 6-year follow-up study

Criteria for exclusion were as follows: alcohol misuse, liver cirrhosis or suspicion of malignancy, chronic viral hepatitis, a history of schisomiasis, anatomical abnormalities in compared to the participants with $\mathrm{FLI}<30$, the adjusted $\mathrm{ORs}(95 \% \mathrm{Cl})$ of
$\mathrm{IVBS}$ were $2.07(1.18-3.62)$ and $2.85(1.39-5.18)$ in the groups of $30 \leq \mathrm{FLI}<60$ adults
and $\mathrm{FLI} \geq 60$, respectively

High FLI scores are an independent risk

In longitudinal analysis, those participants with $\mathrm{FLI} \geq 60$ compared to those with $\mathrm{FLI}<30$ had an increased risk of asymptomatic IVBS [adjusted HR $=1.65$ (95\% Cl: 1.05-2.60)]

vertebrobasilar system, individuals receiving anti-hypertension treatment, those under glucos

Cicero et al. ${ }^{[39]} 20181731$ adult volunteers recruited in the Brisighella study were classified as NASH low-risk (BMI $<28$ and no diabetes), NASH intermediate-risk (BMI $\geq 28$ or diabetes), or NASH high-risk (BM $\geq 28$ and diabetes)

Multiple linear regression analysis was assessed for predictors of AS

The exclusion of people with hypertension, T2D, and MetS did not alter the associations between FLI and asymptomatic IVBS

Among low risk individuals, $\mathrm{HSI}(\mathrm{RR}=0.138,95 \% \mathrm{Cl}: 0.105-0.170, P<0.001), \quad \mathrm{FLI}$ was invariably associated with $\mathrm{AS}$ in FLI (RR $=0.024,95 \% \mathrm{Cl}: 0.016-0.032, P<0.001), \mathrm{LAP}(\mathrm{RR}=0.014,95 \% \mathrm{Cl}$ : subjects with different metabolic risk $0.008-0.020, P<0.001)$, and SUA (RR $=0.150,95 \% \mathrm{Cl}: 0.024-0.275, P=$ 0.019 ) were significant predictors of $A S$ profiles

$\mathrm{HSI}$ and FLI predicted PWV among intermediate NASH risk volunteers (RR = $0.116,95 \% \mathrm{Cl}: 0.071-0.160, P<0.001 ; \mathrm{RR}=0.010,95 \% \mathrm{Cl}: 0.001-0.020, P=$ $0.041)$

In NASH-high risk participants, FLI and SUA were associated with PWV (RR = $0.049,95 \% \mathrm{Cl}: 0.011-0.087, P=0.013 ; \mathrm{RR}=0.632,95 \% \mathrm{Cl}: 0.222-1.041, P=$ $0.003)$

Olubamwo et al. ${ }^{[40]} 1205$ middle-aged men, free of CVD at baseline from the KIHDRFS 2018 cohort, were evaluated

The associations of baseline FLI with incident CVD and incident AMI were analyzed using multivariable-adjusted Cox regression models

$$
\begin{aligned}
& \text { During a median 17-year follow-up, } 690 \text { incident } \\
& \text { of AMI were recorded through Finnish registries }
\end{aligned}
$$

In the minimally adjusted model for incident CVD, the HR was $1.77(95 \% \mathrm{Cl})$ : 1.46-2.14 [for the high $(\geq 60)$ vs. the low $(\leq 30)$ FLI category].

The association was progressively attenuated with increasing adjustment.

In the most adjusted model, the HR was 1.41 (95\%Cl: 1.10-1.79). The HR for incident AMI was 1.65 (95\% Cl: 1.22-2.23) for the high FLI category in the minimally adjusted model, but, in the more comprehensive models when metabolic factors were included, the HR was not significant $(H R=1.136$, 95\% Cl: 0.777-1.662)

Olubamwo et al. ${ }^{[41]} 501$ Finnish men without CMD during the initial 4-year follow-up 2019 in the KIHDRFS cohort were enrolled

Over the initial 4-year follow-up, $26.9 \%$ of individuals had a significant $(\geq 10)$ FLI increase. The association of 4 -year FLI increase with incident CMD was analyzed in multivariableadjusted Cox regression models, adjusting for baseline constitutional and lifestyle factors (Model 1) and, in addition,

Compared to subjects with low baseline FLI and no significant 4-year FLI increase (used as the reference), subjects with intermediate baseline FLI and significant 4 -year $\mathrm{FLI}$ increase, the HRs and $95 \% \mathrm{Cls}$ for incident CMD in Model 1 [2.13 (1.45-3.13)] and Model 2 [1.73 (1.13-2.66)] exceeded values for subjects with similar baseline FLI without a significant 4-year change [HRs (95\%Cls) were 1.36 (0.94-1.97) for Model 1 and 1.18 (0.81-1.70) for Model 2]
Although FLI can predict incidents of CVD, the predictability of AMI using FLI is subject to interactions with metabolic factors

Individuals with FLI scores falling in the moderate to high categories should be evaluated and monitored for either subclinical or overt CVD, including CAD

Significant increases of FLI scores are associated with increasing CMD risk. Such individuals should be evaluated for progressive FLD and CMD and managed to reduce such a risk 
metabolic and inflammation biomarker factors (Model 2)

Roh et al. ${ }^{[42]} 2020$ The association of FLI scores with new-onset HF was evaluated with multivariate Cox proportional-hazards models in 308,578 healthy people without co-morbidities who underwent the National Health checkups in the republic of Korea from 2009 to 2014

3011,588 subjects in the KNHIS cohort without a history of CVD who underwent health examinations from 2009 to 2011 were identified

Primary endpoint: a composite of cardiovascular deaths, non-fata $\mathrm{Ml}$, and ischemic stroke
Iwasaki et al. ${ }^{[44]}$ 2021

Li et al. ${ }^{[45]} 2021$
FLI score was estimated among 2437 Japanese men. Employees of a single construction company submitted to mandatory annual health checkups and who were free of any history of CVD. baPWV was also measured at the beginning of the study and after a 3-year follow-up

The association of FLI with LVM (assessed by two-dimensional guided $\mathrm{M}$-mode echocardiography) and LVMI was prospectively investigated among 1962 participants from BHS (1995-2010) and 1547 participants from YFS (2001-2011) who were CVD-free at baseline
They approached $\mathrm{HRs}(95 \% \mathrm{Cl})$ for subjects who maintained high $\mathrm{FLI}$ over the 4 years [HRs (95\% Cls) were $2.18(1.54-3.10)$ in Model 1 and 1.85 (1.21-2.82) in Model 2]

A total of 2532 subjects $(0.8 \%)$ received a new diagnosis of $\mathrm{HF}$ during the study period (a median of 5.4 years)

Patients were categorized into quartile groups according to FLI scores ( Q1, O4.9; Q2, 5.0-12.5; Q3, 12.6-31.0; and Q4, > 31.0)

The cumulative incidence of $\mathrm{HF}$ was significantly higher in the highest $\mathrm{FL}$ group than in the lowest FLI group [Q1, 307 (0.4\%); Q4, 890 (1.2\%); P< $0.001]$

Adjusted HRs showed that the highest FLI group was independently associated with an increased risk for HF (HR between Q4 and Q1, 2.709; $95 \% \mathrm{Cl}: 2.380-3.085 ; P<0.001)$. FLI was significantly associated with an increased risk of new-onset $\mathrm{HF}$ regardless of baseline characteristics

During the median 6-year follow-up period, there were 46,010 cases of MACEs ( 7148 cases of cardiovascular death, 16,574 of non-fatal MI, and 22,288 of ischemic stroke)

Higher FLI scores were linearly associated with a higher incidence of the primary endpoint

In the multivariable models adjusted for factors such as body weight and cholesterol levels, the HR for the primary endpoint comparing the highest vs. lowest quartiles of the $\mathrm{FLI}$ was 1.99 ( $95 \% \mathrm{Cl}: 1.91-2.07$ ). The corresponding $\mathrm{HR}(95 \% \mathrm{Cls})$ for cardiovascular death, non-fatal $\mathrm{Ml}$, and ischemic stroke were 1.98 (1.9-2.06), 2.16 (2.01-2.31), and 2.01 (1.90-2.13), respectively ( $P<$ $0.001)$

The results were similar when we performed stratified analyses by age, sex lipid lowering agents, obesity, diabetes, and hypertension

FLI was significantly correlated with the baPWV $(r=0.24, P<0.01)$ FLI may be a marker of AS among Japanese delta change of the baPWV during the study period $(r=0.11, P=0.01)$

Sign

Significant and positive associations between FLI and LVM (BHS: $\beta=0.59, P<F L I$ was positively associated with

$0.001 ;$ YFS: $\beta=0.41, P<0.001$ ) and LVMI (BHS: $\beta=0.14, P<0.001 ;$ YFS: $\beta=$ LVM/LVMI, independent of traditional CVR $0.09, P<0.001$ ) were found in both study cohorts

The association of FLI with LVMI was stronger in women than men (BHS: $P$ interaction $=0.01 ;$ YFS: $P$-interaction $<0.01$ ), and the relationship between $\mathrm{FLI}$ and LVM/LVMI was stronger in black than white individuals (LVM: $P$ LVM/LVMI, independent of traditional CVR
factors. However, such relationships, which interaction $=0.02$; LVMI: $P$-interaction $=0.04$ ) are more pronounced among women and black individuals, are attenuated by high

Higher FLI scores were independently associated with increased risk of $\mathrm{HF}$ in a healthy Korean population

FLI has prognostic value for detecting individuals at higher risk for MACEs physical activity 
Both the associations of FLI with LVM and LVMI were attenuated by high physical activity, especially in BHS ( $P$-interaction $=0.02$ )

Zou et al. ${ }^{[46]} 2021$ Population-based retrospective cohort study using the UK Biobank The mean FLI score in the study cohort was 44.9 . Overall, 33.7\% met the database criteria for NAFLD
At baseline, FLI scores were significantly associated with a wide spectrum of CVR factors

During a mean 7.86-year follow-up the combined incidence of CVD was 6.92 per 1000-person years at risk

In the fully adjusted model, FLI was significantly associated with incident CVD

FLI was significantly associated with incident CVD among subsets of patients stratified by either BMI or varying FLI scores $(<30,<60$, and $\geq 60)$

AMI: Acute myocardial infarction; AS: arterial stiffness; baPWV: brachial-ankle pulse wave velocity; BHS: Bogalusa Heart Study; BMI: body mass index; CAD: coronary artery disease; CI: confidence interval; CMD: cardiometabolic disease; CVD: cardiovascular disease; FLD: fatty liver disease; FLI: Fatty Liver Index; HF: heart failure; HR: hazard ratio; HSI: hepatic steatosis index; IVBS: intracranial vertebrobasilar stenosis; KIHDRFS: Kuopio Ischemic Heart Disease Risk Factor Study; KNHIS: Korean national health insurance system; LAP: lipid accumulation product; LVM: left ventricular mass; LVMI: left ventricular mass indexed to body height: MACEs: major adverse cardiovascular events: MetS: metabolic syndrome: NASH: nonalcoholic steatohepatitis: PMMJS: the prevention of metabolic syndrome and multi-metabolic disorders in Jiangsu: RR: relative risk: SUA: serum uric acid; T2D: type 2 diabetes; PWV: pulse wave velocity; YFS: cardiovascular risk in young Finns study.

\section{FLI, PREDIABETES, AND DIABETES}

Robust evidence supports the notion that NAFLD is not only an effect of pre-existent impaired glucose tolerance and T2D but also a precursor of incident T2D and MetS ${ }^{[51-53]}$

Therefore, it is logical to postulate that FLI may also anticipate states of incident impaired glucose tolerance and overt diabetes. Eight studies published thus far have addressed this research question, as summarized in Table $4^{[54-61]}$.

The studies summarized in Table 4 support the conclusion that there is a direct dose-response association between FLI scores and risk of incident T2 $\mathrm{D}^{[59]}$, as FLI $\geq 60$ specifically predicted T2D among men without Met $S^{[57]}$. Consistently, FLI < 30 predicts prediabetes reversal, particularly among individuals with a healthy lifestyle ${ }^{[61]}$. Therefore, in a primary care setting, FLI may screen individuals to be submitted to aggressive intervention to prevent the progression of prediabetes to overt $\mathrm{T} 2 \mathrm{D}^{[56]}$. This is an originally unexpected but logical utilization of FLI based on the pathophysiology of the NAFLD-T2D association ${ }^{[2-64]}$. If FLI is indeed associated with incident T2D, we can postulate that FLI is also able to identify such a link between NAFLD and CKD.

\section{FLI AND CHRONIC KIDNEY DISEASE}

Recently, research and clinical interest has been raised on the independent association of NAFLD with CKD ${ }^{[5]}$. Two published studies thus far have used FLI to 
Table 4. Published studies supporting the notion that FLl is a risk factor of incident diabetes

\section{Author, year [Ref.] Method Findings}

Matsushita et al. ${ }^{[54]} 2021$ 176 T2D patients admitted to Iwate Medical University Hospital Yahaba, Japan, during the period from January 2017 to March 2019 were recruited. Criteria for exclusion were as follows: cancer, infectious diseases, collagen disorders, diabetic ketoacidosis, and advanced CKD (chronic kidney disease)

Serum CXCL14 concentrations were determined by ELISA. They examined the associations of serum CXCL14 levels with laboratory values, abdominal CT image information, and surrogate markers used for evaluating T2D, obesity, and atherosclerosis

Hirata et al. ${ }^{[55]} 2018 \quad 1498$ men and 2941 women participating in specific health checkups in Japan were recruited. These individuals were divided into six groups based on FLI tertiles (low, moderate, and high) and IFG present/absent, by sex

HR were calculated for incident T2D for each group using an adjusted Cox proportional hazard model

Franch-Nadal et al. ${ }^{[56]}$ 2018

FLI was calculated at baseline for 1142 adult subjects with prediabetes attending primary care centers and classified into
three categories: no steatosis $(F L I<30)$, intermediate $(F L I: 30-$ 60) and hepatic steatosis (FLI $\geq 60$ )

The incidence rate of T2D in each FLI category was assessed at 3 years of follow-up and calculated using fully adjusted ${ }^{\star}$ Cox regression models

Olubamwo et al. ${ }^{[57]} 2019$ This prospective study enrolled 1792 Finnish non-diabetic at the baseline in the KIHDRFS cohort

The association of baseline FLD with incident T2D was analyzed in multivariable-adjusted Cox regression models, considering their MetS statuses

The main models were adjusted for constitutional factors, lifestyle factors, biomarkers of inflammation, and FLI categories high $(\geq 60)$ vs. low $(<30)$
CXCL14 serum concentrations were positively associated with BMI, WC,

subcutaneous and visceral fat areas, serum ALT, SUA, total cholesterol, LDLcholesterol, TG, and C-peptide levels. In contrast, CXCL14 levels correlated inversely with age, pulse wave velocity, and serum adiponectin levels

Conclusion

Multiple LRA showed serum levels of $C$-peptide $(\beta=0.227, P=0.038)$ and the $F$ $(\beta=0.205, P=0.049)$ to be the only parameters showing independent statistically significant associations with serum CXCL14 levels

During a mean follow-up period of 3.0 years, 176 cases of T2D in men and 320 cases in women were identified

Compared to the low FLI group-no IFG, the high FLI group no-IFG was significantly associated with incident T2D in both men (HR $=1.90 ; 95 \% \mathrm{Cl}: 1.08-3.36)$ and women $(\mathrm{HR}=1.72 ; 95 \% \mathrm{Cl}: 1.18-2.51)$

All IFG groups were significantly associated with incident T2D irrespective of FL scores

The proportion of subjects with prediabetes and hepatic steatosis $(F L I \geq 60)$ at baseline was $55.7 \%$

The incidence rate of T2D at 3 years follow-up was 1.3, 2.9, and 6.0 per 100 personyears for $\mathrm{FH}<30, \mathrm{FH} 30$ to $<60$, and $\mathrm{FH} \geq 60$, respectively

The most significant variables increasing the risk of developing T2D were MetS (HR $=3.02 ; 95 \% \mathrm{Cl}: 2.14-4.26)$ and $\mathrm{FLI} \geq 60$ ( $\mathrm{HR}=4.52 ; 95 \% \mathrm{Cl}: 2.10-9.72)$. Moreover $\mathrm{FLI} \geq 60$ was independently associated with T2D incidence: the HR was 4.97 (95\%Cl: 2.28-10.80) in the base regression model adjusted for sex, age, an educational level and 3.21 (95\% Cl: 1.45-7.09) in the fully adjusted model

In the full model, the $\mathrm{HR}(95 \% \mathrm{Cl})$ for T2D was 3.68 (2.80-4.82). The association was attenuated, but maintained, with further adjustment for metabolic factors

When adjusted for MetS status instead of metabolic factors, the $\mathrm{HRs}(95 \% \mathrm{Cls})$ were $2.63(1.92-3.59)$ for FH $\geq 60$ and 177 (1.35-2 31) for Mets

In MetS-stratified analysis, FL predicted T2D only among persons without MetS

In unstratified analysis with subjects categorized by FLI-MetS, persons with FLI $\geq 60$ without MetS had increased risk for T2D $[H R=3.19(2.26-4.52)]$ compared to people with $\mathrm{FLI}<30$ without MetS
Serum CXCL14 levels were dependently associated with atients

$\mathrm{FLI}$ is associated with the development of T2D regardless sex and the presence or absence of $I F G$, and it may be a useful predictor of future risk of incident T2D without IFG

In a primary care setting, $\mathrm{FLI}$ is an

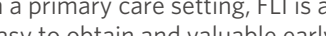
mark of high risk of incident

T2D in patients with priabetes

FLI may allow the identification of individuals to submit to aggressive intervention to prevent and reduce prediabetes progression

$\mathrm{FLI} \geq 60$ specifically predicted T2D among men without MetS but not among men with MetS increases the risk

Both FLI and MetS can mutually complement each other in screening and surveillance of 
Wargny et al. ${ }^{[58]} 2019$ The IT-DIAB study, a 5-year, prospective, observational study carried out in occupational centers based in three French cities, included 389 individuals with prediabetes, defined as FPG $\geq 100$ and $\leq 125 \mathrm{mg} / \mathrm{dL}$. NOD conversion was defined as a first FPG value $\geq 126 \mathrm{mg} / \mathrm{dL}$ and prediabetes reversion as a first FPG value $<110 \mathrm{mg} / \mathrm{dL}$

The associations of both events with baseline FLI were studied separately using multivariate Cox models

Movahedian et al. ${ }^{[59]}$ 2020

Niu et al. ${ }^{[60]} 2021$

This prospective population-based sample cohort of resident from Beiiing and Shanghai included 1781 Chinese aged 50-70 meta-analysis with $\mathrm{DerSim}$ (s) of studies were pooffects models to find combined HRs

The dose-response effect of this relationship was also assessed years and submitted to a 6 -year follow-up

Individuals with $\mathrm{FLI}<30$ and MetS had greater risk $[\mathrm{HR}=4.31(2.15-8.61)]$ and people with both $\mathrm{FLI} \geq 60$ and MetS had the greatest risk $[\mathrm{HR}=4.66(3.42-6.35)]$ After a median follow-up of 3.9 years (range: 0.1-6.1), 138 individuals (35.5\%) converted to NOD. FLI was associated with a higher risk of NOD conversion (unadjusted $\mathrm{HR}$ per $\mathrm{SD}=1.54,95 \% \mathrm{Cl}: 1.27-1.86, P<0.0001$ ), even after multiple adjustment on FPG, $\mathrm{HbA1c}$, and diabetes risk score (adjusted HR per SD 1.31, 95\%C : $1.07-1.61, P=0.008$ ). FLI was also associated with pre-diabetes reversion: adjusted $\mathrm{HR}$ per $\mathrm{SD}=0.85,95 \% \mathrm{Cl}: 0.75-0.96, P=0.0077$. Changes in $\mathrm{FLI}$ were significantly associated with changes in FPG during follow-up $(P<0.0001)$. When compared to a full model including the diabetes risk score, FPG, HbA1c, and FLI, only HbA1c added significant prediction information (AUROC: $72.8 \%$ for full model vs. $69.4 \%$ for the model without $\mathrm{HbA1c} ; P=0.028$ ), while the removal of $\mathrm{FL}$ to the full model did not alter its predictive value (AUROC: $72.2 \%$ ). The predictive value for NOD conversion was not significantly better for HOMA-IR compared to FLI (AUROC: $69.3 \%$ vs. $63.7 \%, P=0.067$

27 studies totaling 70,918 participants were included in the meta-analysis Pooled results show that the highest category of FLI was associated with an increased incidence of T2D (HR $=2.88,95 \% \mathrm{Cl}: 2.18-3.81$; $P$ for heterogeneity: 0.001)

The source of heterogeneity could not be explained by subgroup analysis (sex,

The pooled HR from the random-effects dose-response model indicated a significant association between $\mathrm{FLI}$ scores and risk of T2D incidence (Coef $=0.0239, P=$ 0.001)

At 6-year resurvey, 463 participants developed T2D

After controlling for $\mathrm{HbA1c}, 9$ of the initially identified 43 glycerolipids remaine significant, including 2 DAGs and 7 TAGs, with RRs $(95 \% \mathrm{Cls})$ ranging from 1.16 (1.05-1.27) to 1.23 (1.11-1.36) per SD increment of glycerolipids

However, additional adjustment for FLI largely attenuated these findings [RR $(95 \% \mathrm{Cls})$ were from $0.88(0.81-0.95)$ to $1.10(1.01-1.21)$ ]

Mediation analyses suggested that the FLI explained 12\%-28\% glycerolipids-T2D associations (all $P<0.01$ )

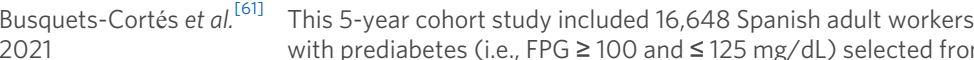
2021 a population of 234,995 potentially suitable individuals who underwent periodic occupational health assessments. Exclusion criteria were diabetes, treatment with oral antidiabetic or systemic glucocorticoid, cancer, anemia, and pregnancy

Prediabetes reversal was defined by FPG $<100 \mathrm{mg} / \mathrm{dL}$

Based on FLI scores, participants were classified as steatosis
At 5 years follow-up, $33.7 \%$ of subjects reverted to normoglycemia (annuat rate of

The adjusted binomial logistic regression model showed that FLI scores $<30$ (OR $1.544 ; 95 \% \mathrm{Cl}: 1.355-1.759)$, performing at least $150 \mathrm{~min} /$ week of physical activity $(\mathrm{OR}=4.600 ; 95 \% \mathrm{Cl}: 4.088-5.177)$ and consuming fruits and vegetables daily $(\mathrm{OR}=$ $1.682 ; 95 \% \mathrm{Cl}: 1.526-1.855)$ were associated with the probability of reverting form prediabetes to normoglycemia

At ROC curve FLI (AUC $=0.774 ; 95 \% \mathrm{Cl}: 0.767-0.781)$ predicted T2D reversal
$\mathrm{FLI}$ is a simple, practical score to further stratify the risk of conversion to NOD or the possibility of prediabetes reversal in clinical practice,

This dose-response metaanalysis supports a direct association between $\mathrm{FLI}$ and $\mathrm{HR}$ of incident T2D

Higher DAGs and TAGs plasma levels were associated with increased risk of incident T2D in this Chinese population. Finding may partially be explained by hepatic steatosis

In adult workers with prediabetes at baseline the independent predictors of prediabetes

reversal to normoglycemia were regular physical activity, healthy hepatic steatosis

$\mathrm{FLI}<30$ predicts the probability of prediabetes reversal, 
*Age, sex, educational level, family history of diabetes, lifestyles, hypertension, lipid profile, and transaminases. ALT: Alanine transaminase; AUC: area under the curve; BMI: body mass index; Cl: confidence interval; CKD: chronic kidney disease; CT: computed tomography; CXCL14: C-X-C motif chemokine ligand 14; DAGs: diacylglycerols; ELISA: enzyme-linked immunosorbent assay; FLD: fatty liver disease; FLI: fatty liver index; FPG: fasting plasma glucose; HbA1c: glycated hemoglobin; HR: hazard ratio; IFG: impaired fasting glucose; KIHDRFS: Kuopio Ischemic Heart Disease Risk Factor Study; LDL: low-density lipoprotein; LRA: linear regression analysis; MetS: metabolic syndrome; NOD: new-onset diabetes; ROC: receiver operator curve; RR: relative risk; SD: standard deviation; SUA: serum uric acid; TAGs: triacylglycerols; TG: triglycerides; T2D: type 2 diabetes; WC: waist circumference.

address this topic, and both seem to confirm the notion that FLI predicts CKD. Sun et al. ${ }^{[65]}$ conducted a population-based study on 9436 adult Chinese subjects. The data show that, in logistic regression analysis, compared to those within the lowest FLI quartile, the adjusted ORs of those in the highest FLI quartile were 2.30 (95\%CI: 1.36-3.90) for increased urinary albumin excretion and 1.93 (95\%CI: 1.18-3.15) for CKD. In the second study, Takahashi et al. . 66$]^{2}$ evaluated the risk of CKD (defined by either estimated glomerular filtration rate $<60 \mathrm{~mL} / \mathrm{min} / 1.73 \mathrm{~m}^{2}$ or positive for urinary protein during a 10-year followup) in 14,163 subjects (male/female: 9077/5086) subjects submitted to annual health examinations. Multivariable Cox regression with restricted cubic spines adjusting for confounders showed that hazard ratios (HRs) of CKD development increased with increasing FLI at baseline in both men and women, and adding FLI to conventional CKD risk factors resulted in a significant improvement in predicting CKD, suggesting that, in a general population cohort study, high FLI scores predict incident CKD in either sex.

\section{FLI AND ENDOCRINE DERANGEMENTS}

NAFLD has also been associated with a variety of endocrine derangements ${ }^{[67-69]}$, some of which predisposing to secondary NAFLD forms, whereas other endocrinopathies probably result from pre-existent NAFLD ${ }^{[70]}$. Does FLI have a role in this setting? Two studies seem to suggest so, although this is a scarcely explored area.

Liu et al. ${ }^{[71]}$, by studying 552 Taiwanese aging men, found that FLI scores were associated with the risk of testosterone deficiency, especially in those without MetS.

Ahn et al. ${ }^{[72]}$, in their study on 4264 Koreans, found a novel nexus linking liver and bone that increases the risk of osteoporosis in men with NAFLD.

Clearly, much research remains to be conducted to ascertain which other endocrinopathies may be associated with FLI scores. 


\section{FLI AND TUMORS}

Various pathomechanisms potentially link NAFLD and various types of tumors, colon adenoma and carcinoma in particular ${ }^{[73]}$. Three studies regarding FLI and tumors have been published thus far, two of them focusing on colorectal adenoma and carcinoma.

In the first study, Ze et al. ${ }^{[74]}$, based on a retrospective observational study on 2976 consecutive $>40$-year-old subjects undergoing routine checkups, found that a high FLI may be useful in predicting colorectal adenoma in relatively healthy Asian populations.

A second study, by Choi et al. ${ }^{[75]}$, was conducted in Korea on data from the National Health Insurance Corporation 2009 to 2012. Although FLI $\geq 60$ was associated with colorectal cancer (CRC) regardless of BMI, the association was more prominent among individuals with a normal BMI. In particular, NAFLD was more closely associated with CRC in the absence of $\mathrm{T} 2 \mathrm{D}$, hypertension, or dyslipidemia than when (one or more of) these conditions were present.

The third study regards FLI and breast cancer. Park et al. ${ }^{[76]}$, using the Korean National Health Insurance Corporation, found that FLI scores of 30-60 and $\geq 60$ were significantly associated with increased breast cancer risk in post-menopausal women hazard ratio ( $\mathrm{HR}=1.07,95 \% \mathrm{CI}$ : $1.04-1.11$; and $\mathrm{HR}=1.11,95 \% \mathrm{CI}$ : 1.05-1.17, respectively), while no such an association was found in pre-menopausal women.

\section{DOES FLI PREDICT MORTALITY?}

Whether FLI is able to assess the risk of death has to be answered cautiously because of the many methodological issues associated with the identification of independent risk factors for mortality ${ }^{[4]}$. Given that NAFLD carries an excess of mortality owing to cardiovascular, cancer, and liver-related causes ${ }^{[77]}$, it is plausible that FLI may be a good marker of increased risk of mortality. Three studies addressed this research question.

Lerchbaum et al. ${ }^{[78]}$, by calculating FLI scores among 3270 subjects submitted to coronary angiography, found that, following a median follow-up time of 7.7 years, patients with high FLI scores compared to those with the lowest FLI scores were independently associated with increased mortality owing to all-causes, cardiovascular causes, and non-cardiovascular causes. The excess risk owing to fatal cancer was of borderline significance.

Based on a median 29-year follow-up of a cohort of 1552 middle-aged men from the Kuopio Ischemic Disease Risk Factor Study, Setti et al. ${ }^{[79]}$ found that those men who had both renal hyperfiltration (RHF) which was associated with smoking - and fatty liver evaluated with FLI scores - which was associated with obesity - had the highest risk of mortality owing to all causes ( $\mathrm{HR}=1.96,95 \% \mathrm{CI}$ : 1.27-3.01). Conversely, having fatty liver associated with normal estimated glomerular filtration rate modestly increased the risk of all-cause mortality ( $\mathrm{HR}=1.35,95 \% \mathrm{CI}$ : 1.09-1.66). Finally, intermediate-risk profiles of all-cause mortality were found among those men who had RHF associated with normal FLI scores. The risk of mortality owing to cardiovascular causes was associated with RHF, rather than with FLI scores. Collectively, the data suggest that RHF and FLI scores are strongly associated with mortality owing to all causes as well as due to cardiovascular causes.

Using a study population of about 3 million individuals submitted to repeated evaluation for health screening purposes over four years, Lee et al. ${ }^{[80]}$ evaluated whether FLI measurements repeated over time could predict incident myocardial infarction (MI), stroke and mortality owing to all causes. They defined 
"FLI points" as the number of times, ranging from zero to four, participants exhibited FLI scores $\geq 60$. This study found that that the higher are the FLI points, the higher is the risk of mortality owing to all causes, MI, and stroke ( $P$ for trend $<0.001$, all). After adjustment for demographic confounders, metabolic cofactor, lifestyle habits, and income, those individuals with four FLI points had a higher risk of mortality owing to all causes $(\mathrm{aHR}=1.86,95 \% \mathrm{CI}: 1.75-1.98, P<0.001)$, incident $\mathrm{MI}(\mathrm{aHR}=1.3,95 \% \mathrm{CI}: 1.21-1.40, P<0.001)$, and incident stroke (aHR $=1.27,95 \% \mathrm{CI}: 1.19-1.37, P<0.001)$. By comparing the first to the last FLI points, the group of individuals with "incident NAFLD" exhibited an increased hazard of mortality compared to the "no NAFLD" group ( $\mathrm{aHR}=1.46,95 \% \mathrm{CI}$ : 1.37-1.55). Consistently, the "regression of NAFLD" group compared to the group with "persistent NAFLD" showed a decreased mortality risk ( $\mathrm{aHR}=0.83,95 \% \mathrm{CI}$ : 0.77-0.89). This study supports the notion that repeating evaluations of FLI scores over time may allow a better profiling of the risks of mortality, MI, and stroke. Moreover, changes of FLI scores over time may help clinicians in evaluating the efficacy of NAFLD treatment and re-modulating prognosis of these patients.

\section{CONCLUSIONS AND RESEARCH AGENDA}

Historically, FLI was proposed in the epidemiological arena as a surrogate index of NAFLD to be used for the identification of cases with suspected NAFLD to be submitted to further ultrasonographic assessment. The data presented in the present SANRA review demonstrate that this primary aim of FLI scores has now been largely overcome by a plethora of other indications. These span all aspects from diagnosis of NAFLD to its (mainly extra-hepatic) manifestations and complications such as atherosclerosis, diabetes, CKD, and tumors.

Importantly, repeating FLI scores over time may allow a non-invasive prediction of overall mortality and serve as a surrogate marker of NAFLD treatment response ${ }^{[8,82]}$ and a useful tool for selecting T2D patients to submit to liver biopsy ${ }^{[83]}$. We expect that the future of FLI will see a further growth in the use of this simple biomarker in several metabolic diseases, NAFLD among them. Conversely, little has been published regarding the ability of FLI to predict liver-related outcomes such as cirrhosis and HCC.

While being based on robust markers of NAFLD pathophysiology, FLI should also be improved by incorporating major modifiers of NAFLD epidemiology, namely age, sex, and reproductive status ${ }^{[84,85]}$, which were originally left out from the FLI algorithm. Of course, the fact that age and sex were left out from the multivariable model which gave birth to the FLI in a single population does not imply that they could not be predictors of NAFLD or other NAFLD-associated outcomes in different populations. The effect of age and sex and other variables of interest can be studied by using them as predictors of a given outcome together with FLI ${ }^{[30]}$.

There are two additional limitations to the use of FLI in clinical practice. The first is the measurement of waist circumference, which, regrettably, tends to be disregarded in the general practice. The second limitation is the "grey zone" of indeterminate FLI scores, which is sex and age dependent and averaged $27.5 \%$ in a recent study ${ }^{[14]}$. The best diagnostic strategy to follow among this substantial proportion of cases remains to identified.

In conclusion, additional studies are eagerly awaited given the importance of FLI as a non-invasive biomarker of NAFLD both in clinical practice and in the research arena. 


\section{DECLARATIONS}

\section{Authors' contributions}

Conceptualization, methodology, software, resources and supervision: Lonardo A, Ballestri S, Bedogni G, Bellentani S, Tiribelli C

Investigation, validation and data curation: Lonardo A

Writing - original draft preparation: Lonardo A

Writing - reviewing and editing: Lonardo A, Ballestri S, Bedogni G, Bellentani S, Tiribelli C

\section{Availability of data and materials}

Not applicable.

\section{Financial support and sponsorship}

None.

\section{Conflicts of interest}

This study was presented by Prof. Stefano Bellentani before the annual Meeting of the Italian Association or the Study of the Liver (AISF) held in Rome, 12th of October 2021 and published in Abstract form in: Digestive and Liver Disease 2021;53:S7-S8.

\section{Ethical approval and consent to participate}

Not applicable.

\section{Consent for publication}

Not applicable.

\section{Copyright}

(c) The Author(s) 2021.

\section{REFERENCES}

1. Lonardo A, Nascimbeni F, Mantovani A, Targher G. Hypertension, diabetes, atherosclerosis and NASH: cause or consequence? $J$ Hepatol 2018;68:335-52. DOI PubMed

2. Lonardo A, Ballestri S. Perspectives of nonalcoholic fatty liver disease research: a personal point of view. Exploration of Medicine 2020;1:85-107. DOI

3. Association for the Study of the Liver (AISF). AISF position paper on nonalcoholic fatty liver disease (NAFLD): updates and future directions. Dig Liver Dis 2017;49:471-83. DOI

4. Ballestri S, Mantovani A, Nascimbeni F, Lugari S, Lonardo A. Extra-hepatic manifestations and complications of nonalcoholic fatty liver disease. Future Med Chem 2019;11:2171-92. DOI PubMed

5. Mantovani A, Petracca G, Beatrice G, et al. Non-alcoholic fatty liver disease and risk of incident chronic kidney disease: an updated meta-analysis. Gut 2020. DOI PubMed

6. Association for the Study of the Liver (EASL), European Association for the Study of Diabetes (EASD), European Association for the Study of Obesity (EASO). EASL-EASD-EASO Clinical Practice Guidelines for the management of non-alcoholic fatty liver disease. Obes Facts 2016;9:65-90. DOI

7. Bedogni G, Bellentani S, Miglioli L, et al. The Fatty Liver Index: a simple and accurate predictor of hepatic steatosis in the general population. BMC Gastroenterol 2006;6:33. DOI PubMed PMC

8. Bedogni G, Miglioli L, Masutti F, Tiribelli C, Marchesini G, Bellentani S. Prevalence of and risk factors for nonalcoholic fatty liver disease: the Dionysos nutrition and liver study. Hepatology 2005;42:44-52. DOI PubMed

9. Lonardo A, Mantovani A, Lugari S, Targher G. Epidemiology and pathophysiology of the association between NAFLD and metabolically healthy or metabolically unhealthy obesity. Ann Hepatol 2020;19:359-66. DOI PubMed

10. Loria P, Marchesini G, Nascimbeni F, et al. Cardiovascular risk, lipidemic phenotype and steatosis. A comparative analysis of cirrhotic and non-cirrhotic liver disease due to varying etiology. Atherosclerosis 2014;232:99-109. DOI PubMed

11. Lonardo A, Lombardini S, Scaglioni F, et al. Hepatic steatosis and insulin resistance: does etiology make a difference? $J$ Hepatol 2006;44:190-6. DOI PubMed

12. Baethge C, Goldbeck-Wood S, Mertens S. SANRA-a scale for the quality assessment of narrative review articles. Res Integr Peer Rev 2019;4:5. DOI PubMed PMC 
13. Weber KS, Simon MC, Strassburger K, et al; GDS Group. Habitual fructose intake relates to insulin sensitivity and Fatty Liver Index in recent-onset type 2 diabetes patients and individuals without diabetes. Nutrients 2018;10:774. DOI PubMed PMC

14. Leone A, Battezzati A, Bedogni G, et al. Sex- and age-related differences in the contribution of ultrasound-measured visceral and subcutaneous abdominal fat to fatty liver index in overweight and obese caucasian adults. Nutrients 2019;11:3008. DOI PubMed PMC

15. Klisic A, Kavaric N, Ninic A. Predictive values of serum uric acid and alanine-aminotransferase for Fatty Liver Index in Montenegrin population. J Med Biochem 2019;38:407-17. DOI PubMed PMC

16. Nivukoski U, Niemelä M, Bloigu A, et al. Combined effects of lifestyle risk factors on fatty liver index. BMC Gastroenterol 2020;20:109. DOI PubMed PMC

17. Tien NV, Arisawa K, Uemura H, Imaeda N, Goto C, Katsuura-Kamano S. Association between nutrient patterns and fatty liver index: baseline survey of the Japan Multi-Institutional Collaborative Cohort Study in Tokushima, Japan. J Epidemiol 2021. DOI PubMed

18. Bertolotti M, Lonardo A, Mussi C, et al. Nonalcoholic fatty liver disease and aging: epidemiology to management. World $J$ Gastroenterol 2014;20:14185-204. DOI PubMed PMC

19. Lonardo A, Suzuki A. Sexual Dimorphism of NAFLD in Adults. Focus on clinical aspects and implications for practice and translational research. J Clin Med 2020;9:1278. DOI PubMed PMC

20. Jensen T, Abdelmalek MF, Sullivan S, et al. Fructose and sugar: a major mediator of non-alcoholic fatty liver disease. $J$ Hepatol 2018;68:1063-75. DOI PubMed PMC

21. Prati D, Taioli E, Zanella A, et al. Updated definitions of healthy ranges for serum alanine aminotransferase levels. Ann Intern Med 2002;137:1-10. DOI PubMed

22. Foschi FG, Conti F, Domenicali M, et al. External validation of surrogate indices of fatty liver in the general population: the Bagnacavallo study. J Clin Med 2021;10:520. DOI PubMed PMC

23. Klisic A, Isakovic A, Kocic G, et al. Relationship between oxidative stress, inflammation and dyslipidemia with Fatty Liver Index in patients with type 2 diabetes mellitus. Exp Clin Endocrinol Diabetes 2018;126:371-8. DOI PubMed

24. Chen LD, Huang JF, Chen QS, et al. Validation of fatty liver index and hepatic steatosis index for screening of non-alcoholic fatty liver disease in adults with obstructive sleep apnea hypopnea syndrome. Chin Med J (Engl) 2019;132:2670-6. DOI PubMed PMC

25. Hsu CL, Wu FZ, Lin KH, et al. Role of Fatty Liver Index and metabolic factors in the prediction of nonalcoholic fatty liver disease in a lean population receiving health checkup. Clin Transl Gastroenterol 2019;10:1-8. DOI PubMed PMC

26. Rabbitt LA, McNally M, Reynolds L, et al. A prospective cohort study of the use of the fatty liver index and Fibroscan to determine the prevalence of fatty liver disease in an Irish population. Eur J Gastroenterol Hepatol 2020. DOI PubMed

27. Chen LW, Huang PR, Chien CH, Lin CL, Chien RN. A community-based study on the application of fatty liver index in screening subjects with nonalcoholic fatty liver disease. J Formos Med Assoc 2020;119:173-81. DOI PubMed

28. Motamed N, Faraji AH, Khonsari MR, et al. Fatty liver index (FLI) and prediction of new cases of non-alcoholic fatty liver disease: a population-based study of northern Iran. Clin Nutr 2020;39:468-74. DOI PubMed

29. Castellana M, Donghia R, Guerra V, et al. Performance of Fatty Liver Index in identifying non-alcoholic fatty liver disease in population studies. a meta-analysis. J Clin Med 2021;10:1877. DOI PubMed PMC

30. Steyerberg EW. Clinical prediction models. Cham: Springer International; 2019.

31. Fedchuk L, Nascimbeni F, Pais R, Charlotte F, Housset C, Ratziu V; LIDO Study Group. Performance and limitations of steatosis biomarkers in patients with nonalcoholic fatty liver disease. Aliment Pharmacol Ther 2014;40:1209-22. DOI PubMed

32. Khang AR, Lee HW, Yi D, Kang YH, Son SM. The fatty liver index, a simple and useful predictor of metabolic syndrome: analysis of the Korea National Health and Nutrition Examination Survey 2010-2011. Diabetes Metab Syndr Obes 2019;12:181-90. DOI PubMed PMC

33. Lee HJ, Jo HN, Kim YH, Kim SC, Joo JK, Lee KS. Predictive value of lipid accumulation product, fatty liver index, visceral adiposity index for metabolic syndrome according to menopausal status. Metab Syndr Relat Disord 2018;16:477-82. DOI PubMed

34. Ballestri S, Lonardo A, Romagnoli D, et al. Ultrasonographic fatty liver indicator, a novel score which rules out NASH and is correlated with metabolic parameters in NAFLD. Liver Int 2012;32:1242-52. DOI PubMed

35. Ballestri S, Nascimbeni F, Lugari S, Lonardo A, Francica G. A critical appraisal of the use of ultrasound in hepatic steatosis. Expert Rev Gastroenterol Hepatol 2019;13:667-81. DOI PubMed

36. Ballestri S, Tana C, Di Girolamo M, et al. Semi-quantitative ultrasonographic evaluation of NAFLD. Curr Pharm Des 2020;26:391527. DOI PubMed

37. Xavier SA, Monteiro SO, Arieira CM, et al. US-FLI score - is it possible to predict the steatosis grade with an ultrasonographic score? Mol Genet Metab 2021;132:204-9. DOI PubMed

38. Qiu J, Zhou Z, Wang J, et al. Associations between fatty liver index and asymptomatic intracranial vertebrobasilar stenosis in Chinese population. PLoS One 2017;12:e187502. DOI PubMed PMC

39. Cicero AFG, Gitto S, Fogacci F, et al; Brisighella Heart Study Group Medical and Surgical Sciences Dept, University of Bologna. Fatty liver index is associated to pulse wave velocity in healthy subjects: data from the Brisighella Heart Study. Eur J Intern Med 2018;53:29-33. DOI PubMed

40. Olubamwo OO, Virtanen JK, Voutilainen A, Kauhanen J, Pihlajamäki J, Tuomainen TP. Association of fatty liver index with the risk of incident cardiovascular disease and acute myocardial infarction. Eur J Gastroenterol Hepatol 2018;30:1047-54. DOI PubMed

41. Olubamwo OO, Virtanen JK, Pihlajamaki J, Mantyselka P, Tuomainen TP. Fatty liver index as a predictor of increased risk of cardiometabolic disease: finding from the Kuopio Ischaemic Heart Disease Risk Factor Study Cohort. BMJ Open 2019;9:e031420. DOI PubMed PMC 
42. Roh JH, Park JH, Lee H, et al. Higher fatty liver index is associated with increased risk of new onset heart failure in healthy adults: a nationwide population-based study in Korea. BMC Cardiovasc Disord 2020;20:204. DOI PubMed PMC

43. Kim JH, Moon JS, Byun SJ, et al. Fatty liver index and development of cardiovascular disease in Koreans without pre-existing myocardial infarction and ischemic stroke: a large population-based study. Cardiovasc Diabetol 2020;19:51. DOI PubMed PMC

44. Iwasaki Y, Shiina K, Matsumoto C, et al. Correlation of the fatty liver index with the pathophysiological abnormalities associated with cardiovascular risk markers in Japanese men without any history of cardiovascular disease: comparison with the fibrosis-4 score. $J$ Atheroscler Thromb 2021;28:524-34. DOI PubMed PMC

45. Li X, Heiskanen JS, Ma H, et al. Fatty liver index and left ventricular mass: prospective associations from two independent cohorts. $J$ Hypertens 2021;39:961-9. DOI PubMed

46. Zou B, Yeo YH, Cheung R, Ingelsson E, Nguyen MH. Fatty liver index and development of cardiovascular disease: findings from the UK Biobank. Dig Dis Sci 2021;66:2092-100. DOI PubMed

47. Ampuero J, Gallego-Durán R, Romero-Gómez M. Association of NAFLD with subclinical atherosclerosis and coronary-artery disease: meta-analysis. Rev Esp Enferm Dig 2015;107:10-6. PubMed

48. Targher G, Byrne CD, Lonardo A, Zoppini G, Barbui C. Non-alcoholic fatty liver disease and risk of incident cardiovascular disease: a meta-analysis. J Hepatol 2016;65:589-600. DOI PubMed

49. Bedogni G, Gastaldelli A, Foschi FG. Fatty liver, cardiometabolic disease and mortality. Curr Opin Lipidol 2020;31:27-31. DOI PubMed

50. Ballestri S, Mantovani A, Baldelli E, et al. Liver fibrosis biomarkers accurately exclude advanced fibrosis and are associated with higher cardiovascular risk scores in patients with NAFLD or viral chronic liver disease. Diagnostics (Basel) 2021;11:98. DOI PubMed PMC

51. Ballestri S, Zona S, Targher G, et al. Nonalcoholic fatty liver disease is associated with an almost twofold increased risk of incident type 2 diabetes and metabolic syndrome. Evidence from a systematic review and meta-analysis. J Gastroenterol Hepatol 2016;31:93644. DOI PubMed

52. Lonardo A, Leoni S, Alswat KA, Fouad Y. History of nonalcoholic fatty liver disease. Int J Mol Sci 2020;21:5888. DOI PubMed PMC

53. Mantovani A, Petracca G, Beatrice G, Tilg H, Byrne CD, Targher G. Non-alcoholic fatty liver disease and risk of incident diabetes mellitus: an updated meta-analysis of 501022 adult individuals. Gut 2021;70:962-9. DOI PubMed

54. Matsushita Y, Hasegawa Y, Takebe N, et al. Serum C-X-C motif chemokine ligand 14 levels are associated with serum C-peptide and fatty liver index in type 2 diabetes mellitus patients. J Diabetes Investig 2021;12:1042-9. DOI PubMed PMC

55. Hirata A, Sugiyama D, Kuwabara K, et al. Fatty liver index predicts incident diabetes in a Japanese general population with and without impaired fasting glucose. Hepatol Res 2018;48:708-16. DOI PubMed

56. Franch-Nadal J, Caballeria L, Mata-Cases M, et al; PREDAPS Study Group. Fatty liver index is a predictor of incident diabetes in patients with prediabetes: the PREDAPS study. PLoS One 2018;13:e0198327. DOI PubMed PMC

57. Olubamwo OO, Virtanen JK, Pihlajamaki J, Tuomainen TP. Association of fatty liver index with risk of incident type 2 diabetes by metabolic syndrome status in an Eastern Finland male cohort: a prospective study. BMJ Open 2019;9:e026949. DOI PubMed PMC

58. Wargny M, Smati S, Pichelin M, et al. Fatty liver index is a strong predictor of changes in glycemic status in people with prediabetes: The IT-DIAB study. PLoS One 2019;14:e0221524. DOI PubMed PMC

59. Movahedian M, Rahmani J, Hashemi Nazari SS, Mohamadi S, Naik G, Hekmatdoost A. Fatty liver index and risk of diabetes incidence: a systematic review and dose-response meta-analysis of cohort studies. Prim Care Diabetes 2020;14:577-83. DOI PubMed

60. Niu Z, Wu Q, Sun L, et al. Circulating glycerolipids, Fatty Liver Index, and incidence of type 2 diabetes: a prospective study among Chinese. J Clin Endocrinol Metab 2021;106:2010-20. DOI PubMed

61. Busquets-Cortés C, Bennasar-Veny M, López-González ÁA, Fresneda S, Abbate M, Yáñez AM. Utility of Fatty Liver Index to predict reversion to normoglycemia in people with prediabetes. PLoS One 2021;16:e0249221. DOI PubMed PMC

62. Samuel VT, Shulman GI. Nonalcoholic fatty liver disease, insulin resistance, and ceramides. N Engl J Med 2019;381:1866-9. DOI PubMed

63. Lonardo A, Lugari S, Ballestri S, Nascimbeni F, Baldelli E, Maurantonio M. A round trip from nonalcoholic fatty liver disease to diabetes: molecular targets to the rescue? Acta Diabetol 2019;56:385-96. DOI PubMed

64. Loomba R, Friedman SL, Shulman GI. Mechanisms and disease consequences of nonalcoholic fatty liver disease. Cell 2021;184:253764. DOI PubMed

65. Sun K, Lin D, Li F, et al. Fatty liver index, albuminuria and the association with chronic kidney disease: a population-based study in China. BMJ Open 2018;8:e19097. DOI PubMed PMC

66. Takahashi S, Tanaka M, Furuhashi M, et al. Fatty liver index is independently associated with deterioration of renal function during a 10-year period in healthy subjects. Sci Rep 2021;11:8606. DOI PubMed PMC

67. Lonardo A, Carani C, Carulli N, Loria P. 'Endocrine NAFLD' a hormonocentric perspective of nonalcoholic fatty liver disease pathogenesis. J Hepatol 2006;44:1196-207. DOI PubMed

68. Loria P, Carulli L, Bertolotti M, Lonardo A. Endocrine and liver interaction: the role of endocrine pathways in NASH. Nat Rev Gastroenterol Hepatol 2009;6:236-47. DOI PubMed

69. Liebe R, Esposito I, Bock HH, et al. Diagnosis and management of secondary causes of steatohepatitis. J Hepatol 2021;74:1455-71. DOI PubMed

70. Lonardo A, Mantovani A, Lugari S, Targher G. NAFLD in some common endocrine diseases: prevalence, pathophysiology, and principles of diagnosis and management. Int J Mol Sci 2019;20:2841. DOI PubMed PMC 
71. Liu CC, Huang SP, Hsieh TJ, et al. Fatty liver index is associated with the risk of testosterone deficiency in aging men without metabolic syndrome. Andrology 2021;9:863-72. DOI PubMed

72. Ahn SH, Seo DH, Kim SH, Nam MS, Hong S. The relationship between fatty liver index and bone mineral density in Koreans: KNHANES 2010-2011. Osteoporos Int 2018;29:181-90. DOI PubMed

73. Lonardo A, Roncucci L. The "obese liver" and gastrointestinal cancer risk. Transl Gastroenterol Hepatol 2020;5:44. DOI PubMed PMC

74. Ze EY, Kim BJ, Jun DH, Kim JG, Kang H, Lee DY. The Fatty Liver Index: a simple and accurate predictor of colorectal adenoma in an average-risk population. Dis Colon Rectum 2018;61:36-42. DOI PubMed PMC

75. Choi YJ, Lee DH, Han KD. Association between high fatty liver index and development of colorectal cancer: a nationwide cohort study with 21,592,374 Korean. Korean J Intern Med 2020;35:1354-63. DOI PubMed PMC

76. Park JH, Choi IS, Han KD, Park H, Kim KH, Kim JS. Association between fatty liver index and risk of breast cancer: a nationwide population-based study. Clin Breast Cancer 2020;20:e450-7. DOI PubMed

77. Liu Y, Zhong GC, Tan HY, Hao FB, Hu JJ. Nonalcoholic fatty liver disease and mortality from all causes, cardiovascular disease, and cancer: a meta-analysis. Sci Rep 2019;9:11124. DOI PubMed PMC

78. Lerchbaum E, Pilz S, Grammer TB, et al. The fatty liver index is associated with increased mortality in subjects referred to coronary angiography. Nutr Metab Cardiovasc Dis 2013;23:1231-8. DOI PubMed

79. Setti M, Voutilainen A, Tuomainen TP. Renal hyperfiltration, fatty liver index, and the hazards of all-cause and cardiovascular mortality in Finnish men. Epidemiol Health 2021;43:e2021001. DOI PubMed PMC

80. Lee CH, Han KD, Kim DH, Kwak MS. The repeatedly elevated fatty liver index is associated with increased mortality: a populationbased cohort study. Front Endocrinol (Lausanne) 2021;12:638615. DOI PubMed PMC

81. Kobyliak N, Abenavoli L, Mykhalchyshyn G, et al. A multi-strain probiotic reduces the Fatty Liver Index, cytokines and aminotransferase levels in NAFLD patients: evidence from a randomized clinical trial. J Gastrointestin Liver Dis 2018;27:41-9. DOI PubMed

82. Drinda S, Grundler F, Neumann T, et al. Effects of periodic fasting on fatty liver index-a prospective observational study. Nutrients 2019;11:2601. DOI PubMed PMC

83. Besutti G, Bonilauri L, Manicardi E, et al. Feasibility and efficiency of European guidelines for NAFLD assessment in patients with type 2 diabetes: A prospective study. Diabetes Res Clin Pract 2021;177:108882. DOI PubMed

84. Lonardo A, Bellentani S, Argo CK, et al; Non-alcoholic Fatty Liver Disease Study Group. Epidemiological modifiers of non-alcoholic fatty liver disease: focus on high-risk groups. Dig Liver Dis 2015;47:997-1006. DOI PubMed

85. Lonardo A, Nascimbeni F, Ballestri S, et al. Sex differences in nonalcoholic fatty liver disease: state of the art and identification of research gaps. Hepatology 2019;70:1457-69. DOI PubMed PMC 